\title{
Effect of methanol extracts of Cnidium officinale Makino and Capsella bursa-pastoris on the apoptosis of HSC-2 human oral cancer cells
}

\author{
KYUNG-EUN LEE ${ }^{1 *}$, JI-AE SHIN $^{2 *}$, IN-SUN HONG $^{3}$, NAM-PYO CHO ${ }^{2}$ and SUNG-DAE CHO ${ }^{2}$ \\ ${ }^{1}$ Department of Oral Medicine, Chonbuk National University; ${ }^{2}$ Department of Oral Pathology, \\ School of Dentistry, Institute of Oral Bioscience, BK21 Project, Chonbuk National University, Jeonju 561-756; \\ ${ }^{3}$ Department of Veterinary Public Health, Laboratory of Stem Cell and Tumor Biology, \\ Seoul National University, Seoul 151-742, Republic of Korea
}

Received November 14, 2012; Accepted December 17, 2012

DOI: $10.3892 /$ etm.2012.871

\begin{abstract}
Cnidium officinale Makino and Capsella bursa-pastoris are used as traditional herbs with diverse medicinal effects, including the inhibition of inflammation, reduction of blood pressure and as diuretics, however, the anticancer effects of $C$. officinale Makino and C. bursa-pastoris are poorly defined. The aims of this study were to evaluate the effects of methanol extracts of $C$. officinale Makino (MECO) and methanol extracts of C. bursa-pastoris (MECB) on the cell growth and apoptosis of HSC-2 human oral cancer cells. $\mathrm{MECO}$ and MECB caused growth inhibition and the induction of apoptosis in a concentration-dependent manner in HSC-2 cells. A marked reduction in specificity protein 1 (Sp1) expression following treatment with MECO or MECB was also observed. The downregulation of $\mathrm{Sp} 1$ by siRNA resulted in growth inhibition and a reduction of total poly (ADP-ribose) polymerase (PARP) expression. In addition, MECO significantly increased Bax expression levels and MECB increased Bak expression levels and decreased Mcl-1 expression levels. These results suggest that MECO and MECB inhibit cell growth and induce apoptosis via the $\mathrm{Sp} 1$ protein, indicating that MECO and MECB are useful bioactive materials and attractive drug candidates for oral cancer.
\end{abstract}

Correspondence to: Professor Sung-Dae Cho, Department of Oral Pathology, School of Dentistry, Institute of Oral Bioscience, BK21 Project, Chonbuk National University, 664-14 Duckjin Dong 1ga, Jeonju 561-756, Republic of Korea

E-mail: efiwdsc@chonbuk.ac.kr

${ }^{*}$ Contributed equally

Key words: Cnidium officinale Makino, Capsella bursa-pastoris, specificity protein 1 , oral cancer, apoptosis

\section{Introduction}

Naturally occurring compounds have attracted considerable attention as cancer chemopreventive agents due to their beneficial effects on human health and their potent anticancer effects (1). Certain naturally occurring compounds have been reported to effectively suppress cell proliferation and tumor progression in in vivo and in vitro experimental models of cancer by inducing apoptosis (2). In particular, naturally occurring compounds derived from plant sources, including curcumin, polyphenols, betulinic acid and ellagic acid, have been studied in various models as modulators of proliferation, angiogenesis, apoptosis and inflammation (3). It has also been demonstrated that the antitumor activities of naturally occurring compounds are associated with the regulation of numerous molecular targets, including p53, VEGF, STAT3, MAPK and PI3K/AKT signaling pathways $(1,3)$. Therefore, it is important to understand the antitumor effects and molecular mechanisms of naturally occurring compounds for chemoprevention and chemotherapy.

Specificity protein $1(\mathrm{Sp} 1)$ is a transcription factor which binds GC/GT-rich promoter elements via three $\mathrm{Cys}_{2} \mathrm{His}_{2}$-type zinc fingers and plays key roles in tumorigenesis $(4,5)$. Sp1 regulates several cancer associated genes associated with the cell cycle, proliferation, differentiation and apoptosis (6). In addition, $\mathrm{Spl}$ is overexpressed in several cancers and is closely correlated with the prognosis of patients (7-9). Notably, naturally occurring compounds, such as curcumin and betulinic acid, have been reported to suppress tumor growth via the downregulation of $\mathrm{Sp} 1$ expression in prostate and bladder cancer cells $(10,11)$. Isorhapontigenin has also demonstrated an anticancer effect by inducing apoptosis through the downregulation of the Sp1/XIAP pathway (12). Therefore, the downregulation of $\mathrm{Sp} 1$ by naturally occurring compounds may be a potential chemopreventive and chemotherapeutic strategy for cancer.

The present study demonstrates that methanol extracts of C. officinale Makino (MECO) and C. bursa-pastoris (MECB) decrease cell growth and induce apoptosis via the downregulation of Sp1 in HSC-2 human oral cancer cells. 


\section{Materials and methods}

Chemicals and antibodies. MECO and MECB were provided by Professor Ki-Han Kwon (Kwangju University, Kwangju, Korea). The DC Protein Assay kit was acquired from Bio-Rad Laboratories Inc., (Madison, WI, USA). Sp1 and actin antibodies were obtained from Santa Cruz Biotechnology (Santa Cruz, CA, USA). Poly (ADP-ribose) polymerase (PARP) antibody was provided by BD Pharmingen ${ }^{\mathrm{TM}}$ (San Jose, CA, USA). Antibodies against Bak, Bax, Bcl-xL and Mcl-1 were supplied by Cell Signaling Technology (Charlottesville, VA, USA).

Cell culture and chemical treatment. HSC-2 human oral cancer cells were provided by Hokkaido University (Hokkaido, Japan). Cells were maintained in DMEM supplemented with $10 \%$ fetal bovine serum and $1 \%$ penicillin/streptomycin at $37^{\circ} \mathrm{C}$ in a $5 \% \mathrm{CO}_{2}$ incubator. The cells were treated with DMSO or various concentrations $(300,600$ and $900 \mu \mathrm{g} / \mathrm{ml})$ of MECO or MECB for 24 or $48 \mathrm{~h}$.

MTS assay. The effects of MECO and MECB on cell growth were investigated using the CellTiter $96^{\circledR}$ Aqueous One Solution Cell Proliferation Assay kit (Promega, Madison, WI, USA). Cells were seeded in 96-well plates and treated with MECO or MECB for 24 or $48 \mathrm{~h}$. MTS solution was added to each well and incubated for $2 \mathrm{~h}$ at $37^{\circ} \mathrm{C}$. The absorbance was measured using an ELISA microplate reader (Bio-Tek Instruments, Inc., Madison, WI, USA) at 490 and $690 \mathrm{~nm}$ (as a blank control).

Detection of nuclear morphological changes. The effects of MECO and MECB on nuclear morphological change were confirmed using the fluorescent nuclear dye, DAPI (Sigma, St. Louis, MO, USA). HSC-2 cells were seeded and treated with MECO or MECB for $48 \mathrm{~h}$. The cells were harvested by trypsinization, resuspended in PBS and then fixed in 100\% methanol at room temperature for $10 \mathrm{~min}$. The cells were deposited on slides and stained with DAPI solution $(2 \mu \mathrm{g} / \mathrm{ml})$. The cell morphological change was observed using a fluorescence microscope equipped with a suitable filter for the DAPI fluorescent dye.

Western blot analysis. The protein concentration of the supernatant was determined using the DC Protein Assay kit. The samples containing equal amounts of protein were resolved by SDS-PAGE and transferred to Immun-Blot PVDF membranes (Bio-Rad Laboratories, Hercules, CA, USA). The membranes were blocked with 5\% skimmed milk in TBST at room temperature for $2 \mathrm{~h}$ and probed overnight at $4^{\circ} \mathrm{C}$ with various primary antibodies. They were then incubated with HRP-conjugated secondary antibodies. After $2 \mathrm{~h}$, the membranes were washed and detected using the ECL Western Blotting Luminol reagent (Santa Cruz Biotechnology).

RNA interference. ON-TARGETplus SMARTpool siRNA sequences targeting $\mathrm{Sp} 1$ and non-targeting control were supplied by Dharmacon Research (Lafayette, CO, USA). The HSC-2 cells were seeded in 6-well plates and transiently transfected with $25 \mathrm{nM}$ siRNA using a DharmaFECT2 transfection

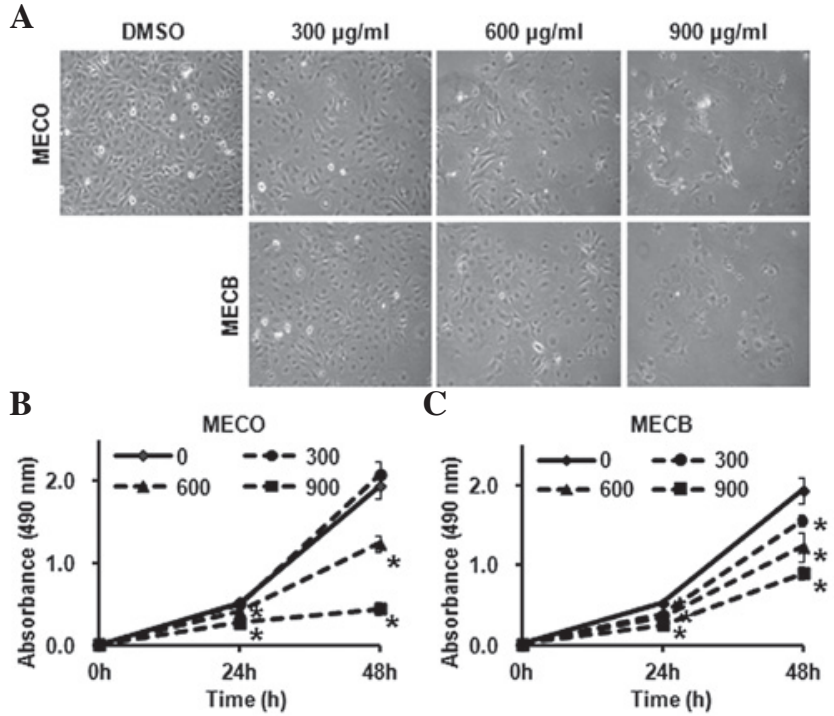

Figure 1. Growth inhibitory effects of MECO and MECB in HSC-2 cells (A) HSC-2 cells were treated with DMSO or various concentrations (300, 600 and $900 \mu \mathrm{g} / \mathrm{ml}$ ) of MECO or MECB for 24 or $48 \mathrm{~h}$. The cell morphology was observed by optical microscope after $48 \mathrm{~h}$, magnification, x200. (B) The effects of MECO and MECB on cell growth were examined using an MTS assay. The results of the inhibitory rates of cell growth were determined from the absorbance at $490 \mathrm{~nm}$. The mean absorbance values are representative of the relative number of viable adherent cells. ${ }^{*} \mathrm{P}<0.05$ compared with the DMSO-treated group. MECO, methanol extracts of $C$. officinale Makino; MECB, methanol extracts of C. bursa-pastoris.

reagent (Thermo Scientific, Lafayette, CO, USA). After 48 h, transfected cells were harvested and examined by trypan blue exclusion assay and western blot analysis.

Trypan blue exclusion assay. The HSC- 2 cells were transfected with $25 \mathrm{nM}$ siRNA for $48 \mathrm{~h}$ and the number of viable cells was counted using a hemocytometer with trypan blue $(0.4 \%)$. The result was expressed as the mean \pm standard deviation.

Statistical analysis. A Student's t-test was used to determine the significance of differences between the control and treatment groups. $\mathrm{P}<0.05$ was considered to indicate a statistically significant result.

\section{Results}

MECO and MECB inhibit cell growth and induce apoptosis in HSC-2 human oral cancer cells. To investigate the anticancer effects of MECO and MECB on HSC-2 human oral cancer cells, we assessed the growth inhibitory effects of MECO and MECB. Cells were treated with DMSO or various concentrations $(300,600$ and $900 \mu \mathrm{g} / \mathrm{ml})$ of MECO or MECB for 24 or $48 \mathrm{~h}$. Morphological changes of the MECO- and MECB-treated cells were observed under an optical microscope after $48 \mathrm{~h}$. As shown in Fig. 1A, a number of MECO- and MECB-treated cells were detached in the medium in a concentration-dependent manner. The effects of MECO and MECB on cell viability were examined using an MTS assay. The results showed that MECO and MECB significantly decreased cell viability in HSC-2 cells (Fig. 1B and C). We then evaluated whether the growth inhibitory effects of MECO and MECB were associated with 
A

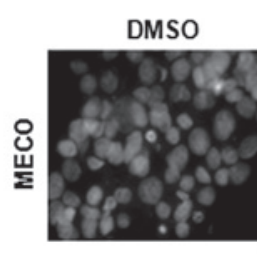

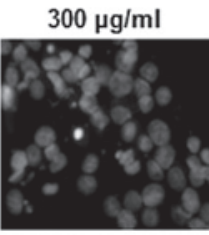
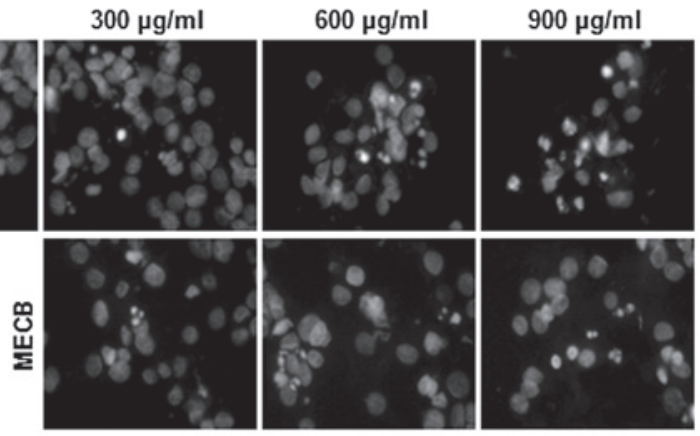

B

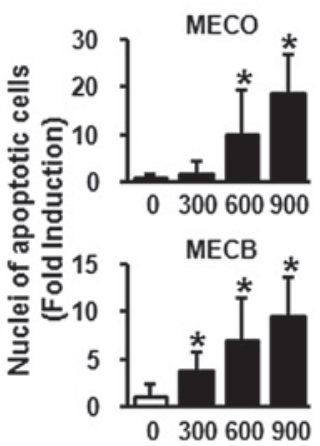

Figure 2. Apoptotic effects of MECO and MECB on HSC-2 cells. (A) HSC-2 cells were treated with DMSO or various concentrations ( 300,600 and $900 \mu \mathrm{g} / \mathrm{ml})$ of MECO or MECB for $48 \mathrm{~h}$. The apoptotic effects of MECO and MECB were determined by DAPI staining. The DAPI-stained cells were observed by fluorescence microscopy, magnification, $\mathrm{x} 400$. (B) The apoptotic cells were counted and expressed as the mean \pm standard deviation. * $\mathrm{P}<0.05$ compared with the DMSO-treated group. MECO, methanol extracts of C. officinale Makino; MECB, methanol extracts of $C$. bursa-pastoris.

A

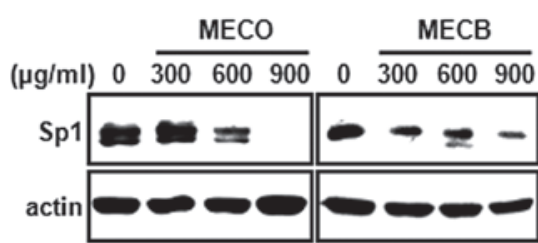

B

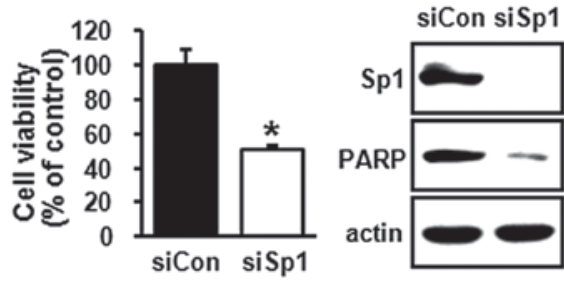

Figure 3. Effects of MECO and MECB on Sp1 expression. (A) HSC-2 cells were treated with DMSO or various concentrations $(300,600$ and $900 \mu \mathrm{g} / \mathrm{ml}$ ) of MECO or MECB for $48 \mathrm{~h}$. The effects of MECO and MECB on Sp1 expression were analyzed by western blot analysis. (B) HSC-2 cells were transfected with siRNA (siCon or siSpl) for $48 \mathrm{~h}$. The cell viability was estimated by trypan blue exclusion assay and whole-cell lysates were analyzed by western blot analysis using antibodies against Sp1, PARP and actin. MECO, methanol extracts of $C$. officinale Makino; MECB, methanol extracts of $C$. bursa-pastoris; $\mathrm{Sp1}$, specificity protein 1; PARP, poly (ADPribose) polymerase.

apoptotic effects using DAPI staining. As shown in Fig. 2A and B, cells treated with MECO or MECB for $48 \mathrm{~h}$ exhibited nuclear fragmentation and chromatin condensation in a concentration-dependent manner. These results demonstrate that MECO and MECB inhibited cell growth and induced apoptosis in HSC-2 human oral cancer cells.

Downregulation of Spl by MECO and MECB correlates with the regulation of several Bcl-2 family proteins. A previous study has suggested that the downregulation of Sp1 inhibits malignant transformation via the induction of apoptosis (13). Since Sp1 is highly expressed in oral tumor tissues compared with normal oral tissues (14), we investigated whether MECO and MECB affect Sp1 expression in HSC-2 cells. The results showed that MECO and MECB decreased Sp1 expression (Fig. 3A). To confirm that the downregulation of Sp1 was associated with the induction of apoptosis, we knocked

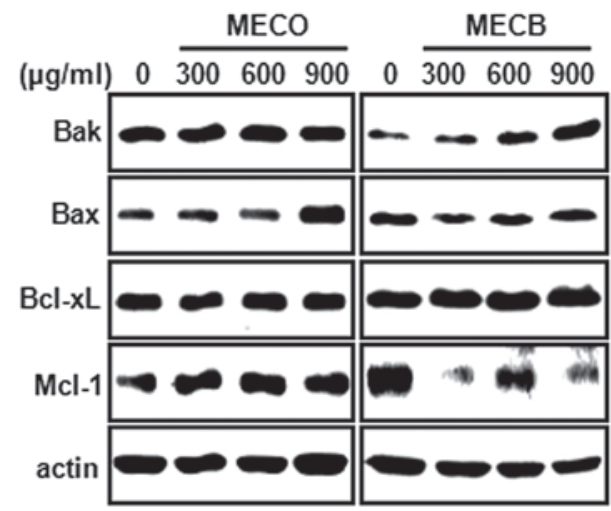

Figure 4. Effects of MECO and MECB on expression of Bcl-2 family proteins HSC-2 cells were treated with DMSO or various concentrations $(300,600$ and $900 \mu \mathrm{g} / \mathrm{ml}$ ) of MECO or MECB for $48 \mathrm{~h}$. The expression of Bcl-2 family proteins was detected by western blot analysis using antibodies against Bak, Bax, Bcl-xL and Mcl-1. MECO, methanol extracts of C. officinale Makino; MECB, methanol extracts of C. bursa-pastoris.

down the expression of Sp1 in the HSC-2 cells using siRNA technology. As shown in Fig. 3B, the knockdown of Sp1 markedly decreased cell growth and total PARP expression, indicating that downregulation of Sp1 was sufficient to inhibit cell growth and induce apoptosis. To further investigate the molecular mechanism of MECO- and MECB-induced apoptosis, we examined whether MECO and MECB regulated the expression of Bcl- 2 family proteins. Fig. 4 shows that $\mathrm{MECO}$ caused a marked increase in Bax expression levels. MECB increased Bak expression and decreased Mcl-1 expression. These results indicate that MECO and MECB may induce apoptosis through the regulation of several Bcl-2 family proteins.

\section{Discussion}

Since $\mathrm{Sp} 1$ is a critical transcription factor which regulates several cancer associated genes associated with cell survival, proliferation and angiogenesis, the abnormal expression or increased binding activity of Sp1 may contribute to tumor development and progression. In a previous study, the abnormally activated $\mathrm{Spl}$ expression was shown to represent a 
potential risk for poor prognosis and directly caused gastric cancer progression (15). The expression of Sp1 in breast cancer tissues is positively associated with TNM stage, tumor invasion and lymph node metastasis (16). In addition, the overexpression of $\mathrm{Sp} 1$ is involved in the malignant transformation of human fibroblasts (13). Previous studies have also evaluated different approaches for targeting Sp1, including Sp1 ribozyme, siRNA and dominant negative mutant, in experimental models. The downregulation of Sp1 by Sp1 ribozyme correlated with increased apoptosis (13), and the silencing of Sp1 by siRNA suppressed invasion in human glioma cells (17). The dominant negative mutant of Sp1 demonstrated a growth inhibitory effect in cervical cancer cell lines (18). Therefore, it is likely that $\mathrm{Sp1}$ is an important target for cancer therapy.

A recent study in our laboratory has shown that $\mathrm{Sp1}$ is overexpressed in oral cancer tissues compared with normal oral tissues (14). Notably, several naturally occurring compounds decreased the cell growth of oral cancer cells, and exhibited apoptotic activity through the decreased expression of $\mathrm{Sp1}$ and regulation of its downstream target proteins $(14,19,20)$. These findings demonstrate that certain naturally occurring compounds regulate $\mathrm{Sp} 1$ expression to inhibit cell growth and induce apoptosis in oral cancer.

In the present study, we examined whether MECO and MECB inhibit cell growth and induce apoptosis through the downregulation of Sp1 in oral cancer cells. We observed that MECO and MECB significantly decreased cell growth and induced apoptosis, which was caused by Sp1 downregulation.

The Bcl-2 family of proteins have emerged as important regulators in mitochondria-mediated apoptosis due to protein-protein interactions between pro- and anti-apoptotic proteins (21). In particular, it is noteworthy that promoters of Bcl-2 family genes such as Mcl-1 and Bax contain Sp1 binding sites $(14,22)$. Therefore, we hypothesized that the downregulation of Sp1 by MECO and MECB may affect pro- or anti-apoptotic proteins. We observed that MECO significantly increased Bax expression, but did not change other Bcl-2 family proteins. MECB markedly increased Bak expression and decreased Mcl-1 expression. Although the effects of MECO and MECB on several Bcl-2 family proteins due to the downregulation of $\mathrm{Sp} 1$ are unclear, the regulation of $\mathrm{Bcl}-2$ family proteins by MECO and MECB may be due, in part, to the effect of MECO and MECB on Sp1 expression.

In conclusion, the results of the present study indicate that MECO and MECB treatment inhibited cell growth and induced apoptosis via the downregulation of Sp1 in HSC-2 human oral cancer cells. These effects involved the regulation of Bcl-2 family proteins, including Bax, Bak and Mcl-1. Therefore, we provide experimental evidence to indicate that MECO and MECB may be attractive anticancer drug candidates targeting $\mathrm{Sp} 1$ in oral cancers.

\section{Acknowledgements}

This study was supported by the Basic Science Research Program through the National Research Foundation of Korea (NRF) funded by the Ministry of Education, Science and Technology (2012001497 and 2012003731).

\section{References}

1. Fulda S: Modulation of apoptosis by natural products for cancer therapy. Planta Med 76: 1075-1079, 2010.

2. Nobili S, Lippi D, Witort E, et al: Natural compounds for cancer treatment and prevention. Pharmacol Res 59: 365-378, 2009.

3. Amin AR, Kucuk O, Khuri FR and Shin DM: Perspectives for cancer prevention with natural compounds. J Clin Oncol 27: 2712-2725, 2009.

4. Safe S and Abdelrahim M: Sp transcription factor family and its role in cancer. Eur J Cancer 41: 2438-2448, 2005.

5. Black AR, Black JD and Azizkhan-Clifford J: Sp1 and krüppel-like factor family of transcription factors in cell growth regulation and cancer. J Cell Physiol 188: 143-160, 2001.

6. Sankpal UT, Goodison S, Abdelrahim M and Basha R: Targeting Sp1 transcription factors in prostate cancer therapy. Med Chem 7: 518-525, 2011.

7. Zhang J, Zhu ZG, Ji J, et al: Transcription factor Sp1 expression in gastric cancer and its relationship to long-term prognosis. World J Gastroenterol 11: 2213-2217, 2005.

8. Wang LW, Li Q, Hua ZL, et al: Expression of transcription factor $\mathrm{Sp} 1$ in human gastric cancer tissue and its correlation with prognosis. Zhonghua Zhong Liu Za Zhi 29: 107-111, 2007 (In Chinese).

9. Jia Z, Gao Y, Wang L, Li Q, et al: Combined treatment of pancreatic cancer with mithramycin $\mathrm{A}$ and tolfenamic acid promotes Sp1 degradation and synergistic antitumor activity. Cancer Res 70: 1111-1119, 2010.

10. Chintharlapalli S, Papineni S, Ramaiah SK and Safe S: Betulinic acid inhibits prostate cancer growth through inhibition of specificity protein transcription factors. Cancer Res 67: 2816-2823, 2007.

11. Chadalapaka G, Jutooru I, Chintharlapalli S, et al: Curcumin decreases specificity protein expression in bladder cancer cells. Cancer Res 68: 5345-5354, 2008.

12. Fang Y, Yu Y, Hou Q, et al: The Chinese herb isolate isorhapontigenin induces apoptosis in human cancer cells by down-regulating overexpression of antiapoptotic protein XIAP. J Biol Chem 287: 35234-35243, 2012.

13. Lou Z, O'Reilly S, Liang H, et al: Down-regulation of overexpressed sp1 protein in human fibrosarcoma cell lines inhibits tumor formation. Cancer Res 65: 1007-1017, 2005.

14. Shin JA, Kim JJ, Choi ES, et al: In vitro apoptotic effects of methanol extracts of Dianthus chinensis and Acalypha australis L. targeting specificity protein 1 in human oral cancer cells. Head Neck: June 25, 2012 (Epub ahead of print).

15. Wang L, Wei D, Huang S, et al: Transcription factor Sp1 expression is a significant predictor of survival in human gastric cancer. Clin Cancer Res 9: 6371-6380, 2003.

16. Wang XB, Peng WQ, Yi ZJ, Zhu SL and Gan QH: Expression and prognostic value of transcriptional factor sp1 in breast cancer. Ai Zheng 26: 996-1000, 2007 (In Chinese).

17. Guan H, Cai J, Zhang N, et al: Spl is upregulated in human glioma, promotes MMP-2-mediated cell invasion and predicts poor clinical outcome. Int J Cancer 130: 593-601, 2012.

18. Chen F, Zhang F, Rao J and Studzinski GP: Ectopic expression of truncated Sp1 transcription factor prolongs the S phase and reduces the growth rate. Anticancer Res 20: 661-667, 2000.

19. Shin JA, Shim JH, Choi ES, et al: Chemopreventive effects of synthetic C-substituted diindolylmethanes originating from cruciferous vegetables in human oral cancer cells. Eur J Cancer Prev 20: 417-425, 2011.

20. Shin JA, Shim JH, Jeon JG, et al: Apoptotic effect of Polygonum Cuspidatum in oral cancer cells through the regulation of specificity protein 1. Oral Dis 17: 162-170, 2011.

21. van Delft MF and Huang DC: How the Bcl-2 family of proteins interact to regulate apoptosis. Cell Res 16: 203-213, 2006.

22. Schmidt T, Korner K, Karsunky H, et al: The activity of the murine Bax promoter is regulated by $\mathrm{Sp} 1 / 3$ and E-box binding proteins but not by p53. Cell Death Differ 6: 873-882, 1999 . 